\title{
Evaluation of five algorithms in predicting the sublocalisation of right ventricular outflow tract arrhythmia (RVOTA) when compared to 3D electroanatomical mapping origin
}

\author{
Raluca Sirbu Prisecaru ${ }^{1 *}\left(\mathbb{0}\right.$, Cristina Leatu $^{1}$, Leila Riahi ${ }^{2}$ and Victor Costache $^{3}$
}

\begin{abstract}
Purpose: To compare the predictive accuracy of five different algorithms as verified by successful ablation site using 3D electroanatomical non-contact mapping in patients with symptomatic and asymptomatic but high ventricular burden RVOT tachycardias.

Methods: 28 Consecutive patients admitted for radiofrequency catheter ablation for symptomatic and asymptomatic, but high ventricular burden idiopathic VPC were recruited for this study. All patients had previous failed or intolerant to beta-blocker and/or at least one class IC anti-arrhythmic agents, and they had normal left ventricular ejection fraction. All patients had documented monomorphic VPC with left bundle branch block morphology and an inferior axis. Concordance of the arrhythmia origin based on ECG algorithm and 3D mapping system site were further evaluated. Of the five algorithms, two algorithms with easy-applicability and having a memorable design (Dixit and Joshi) and three algorithms with more complex and detailed design (Ito, Zhang, Pytkowski) were selected for comparisons.

Results: Assessment of the diagnostic accuracy showed that each of the five algorithms had only moderate accuracy, and the greatest accuracy was observed in the algorithm proposed by Pytkowski algorithm when assessed by a general cardiologist and Dixit algorithm when evaluated by the electrophysiologist. However, when the algorithms were compared for their accuracy, specificity, sensitivity, no significant differences were found $(p=0.99)$.

Conclusions: The ECG based algorithms for precise localising RVOTA origin simplify the mapping process, reduce the procedural and fluoroscopic time, and improve clinical outcomes, resulting in greater clinical utility. All the five published 12-lead ECG algorithms for ROTVA differentiation were similar in terms of the diagnostic accuracy, specificity, sensitivity and LRs.
\end{abstract}

Keywords: Right ventricular outflow tract origin, Ventricular premature beats, Algorithms, Radiofrequency ablation, Left bundle branch block morphology

\section{Introduction}

The majority of idiopathic ventricular arrhythmias arise from the right ventricular outflow tract (RVOT) [1], and

\footnotetext{
*Correspondence: raluvsirbu@gmail.com

${ }^{1}$ Cardiology Department, European Polisano Medlife Hospital, Izvorului Street 1A, 550172 Sibiu, Romania

Full list of author information is available at the end of the article
}

they represent nearly $10 \%$ of all ventricular tachycardia (VT) admissions [2, 3]. Frequent premature ventricular complexes (PVCs) or non-sustained and sustained ventricular tachycardias are among the most common presentation scenarios, and they may cause heart failure and ventricular dysfunctions [4]. The question of how many PVCs are required to cause PVC-induced 
cardiomyopathy is not yet completely answered. Though, in one study from Carballeira et al. [5], the PVC burden was not associated with the development of cardiomyopathy (a PVC burden higher than 10\% being used as an inclusion criterion in this study), several other studies showed correlation between PVC frequency and cardiomyopathy [6, 7]. The results of Baman et al's study revealed that a PVC burden higher than $24 \%$ best-differentiated patients is likely to develop an impaired left ventricular (LV) function from those less likely to develop dysfunction. Also, patients with preserved left ventricular function but dilated LV were associated with an intermediate PVC burden of $22 \%$, which was significantly lower compared to patients with LV systolic dysfunction but significantly higher compared to patients with normal LV dimensions and systolic function [7].

PVCs originating from the RVOT are characterised by left bundle branch block (LBBB) morphology on 12 lead electrocardiograms (ECGs) with an inferior frontal plan axis. Most frequently, the site of origin of those tachycardias is established to be in the anterior septal region below the pulmonary valve [8]. However, less frequently the origin site is found in the right ventricular free wall, posterior septal area and other rare areas [9-11].

In patients with symptomatic RVOT-VT/PVCs, primary catheter ablation is recommended [12]. In published reports, acute success rates of RVOT-VT/PVC catheter ablation are $>95 \%$ in patients without structural heart disease [13-15]. Therefore, it is paramount to precisely predict the origin of RVOT arrhythmias to define the appropriate approach before the ablation procedure. Conventional 12-lead electrocardiogram (ECG) is a useful tool for analysing cardiac arrhythmias, and numerous ECG algorithms for predicting the origin of RVOT arrhythmias have been reported [16-25].

The aim of this study was to compare the predictive accuracy of five different algorithms as verified by successful ablation site using 3D electroanatomical noncontact mapping in patients with symptomatic and asymptomatic but high ventricular burden RVOT tachycardias. Of the five algorithms, two algorithms having easy applicability and a memorable design (Dixit [26] and Joshi [3]) and three more complex algorithms of a more detailed design (Ito [12], Zhang [24], Pytkowski [27]) were selected for comparisons.

\section{Algorithms}

1. The Ito algorithm (2003) is based on evaluating the $S$ wave amplitude in $\mathrm{V} 6$ and the determination of transition zone in V4 or S wave in lead I. Furthermore, the $\mathrm{R} / \mathrm{S}$ amplitude index, the $\mathrm{R}$ duration index and the QRS pattern in lead I are determined. Finally, the QRS morphology in aVL, aVR, lead I and inferior leads, and the $\mathrm{S}$ wave in $\mathrm{V} 2$ are evaluated. Three arrhythmia areas in the RV (septal, free wall, near His, left ventricular endocard/epicard, and left coronary sinus) are defined.

2. The Dixit algorithm (2003) uses "notching" in leads II, III, and aVF, and late precordial transition $(R \geq S$ at or beyond lead $\mathrm{V} 4$ ) to predict the arrhythmia origin. QRS polarity in lead aVL was assessed to determine the anterior or posterior arrhythmia locations.

3. The Joshi and Wilber scheme (2005) is based on three criteria for separating the septal from the free wall sites (QRS duration $\geq 140 \mathrm{~ms}$, R-wave "notching" in inferior leads, lead V3 R/S ratio $\leq 1$ ). Then, according to the QRS polarity in lead I, anterior or posterior location of arrhythmias is established. Finally, the QRS polarity in aVL lead determines caudal or cranial sites.

4. The Zhang algorithm (2009) starts with the determination of the QRS morphology and axis. Arrhythmia origin is located in four different sites (near the His, left ventricular origin, RV septal or free wall origin). $\mathrm{RV}$ arrhythmia origins are defined as anterior, posterior and mid regions. LBBB morphology with inferior axis suggested RVOT origin. Then, transitional zone in V4 is determined. Later on, the $\mathrm{R}$ wave duration index, $\mathrm{R} / \mathrm{S}$ amplitude index and $\mathrm{RR}^{\prime} / \mathrm{RSR}^{\prime}$ pattern in lead $\mathrm{aVL}$ are evaluated. Afterwards, PVC-QRS duration/the preceding sinus beat QRS duration and QRS pattern in lead I are evaluated. R duration index and precordial leads transitional zone are determined.

5. The Pytkowski scheme (2013) uses "notching" in leads II, III, and aVF and lower R wave amplitude in these leads to predict free wall origin. Based on the lead I polarity, RVOT is divided into three vertical zones (posterior to anterior). Then, depending on the transitional zone in the precordial leads, three horizontal zones were defined, with horizontal intermediate zone $\left(\mathrm{R}[\mathrm{r}]=\mathrm{S}[\mathrm{s}]\right.$ in $\left.\mathrm{V}_{4}\right)$ occupying a small region of the median RVOT part and separating superior and inferior zones. Three vertical and three horizontal zones divided RVOT into nine subregions.

\section{Methods}

\section{Study population}

Twenty-eight consecutive patients admitted in our centre for radiofrequency catheter ablation of idiopathic PVC between November 2016 and February 2021 were included in this study. All patients had documented monomorphic PVC with left bundle branch block morphology and an inferior axis. Although symptomatic patients usually have a high PVC burden $(>10,000$ 
PVCs/24 h), symptoms are not exclusive to these patients and those with a reduced PVC burden (5000$10,000 / 24$ h) may also be highly symptomatic and warrant ablation [28]. For asymptomatic patients with a high volume of PVCs, prophylactic catheter ablation may be proposed to prevent LV dysfunction and cardiomyopathy. The cut-off is still not yet defined, but several studies have demonstrated that a cut-off value of $20-24 \%$ PVCs per $24 \mathrm{~h}$ is associated with an increased risk of developing reduced LV function and cardiomyopathy $[6,7]$.

In our study, both symptomatic patients with PVCs refractory or intolerant to antiarrhythmic drug (betablocker and/or at least one class IC anti-arrhythmic agents) with a ventricular burden $>5 \%$ and asymptomatic patients with ventricular burden $>20 \%$ on a $24-\mathrm{h}$ Holter monitoring were included. All patients had normal left ventricular ejection fraction.

All the ECG recordings were analysed at a paper speed of $25 \mathrm{~mm} / \mathrm{s}$ and $50 \mathrm{~mm} / \mathrm{s}$. All ECGs were reviewed by one cardiologist following each algorithm and next by one electrophysiologist to establish the origin of the arrhythmia. Concordance of the arrhythmia origin based on the ECG algorithm and 3D mapping system site was further evaluated.

To determine the correct sublocalisation of RVOT arrhythmias (RVOTA) origin, several criteria were established:

1. For the Ito algorithm using two approximate locations in RVOT-septal or free wall, correct sublocalisation was identified if ECG-based origin corresponded with the EPS origin.

2. For the Dixit and Zhang algorithms using 4-6 RVOT zones-septal (anterior, mid, posterior) and free wall (anterior, mid, posterior), correct sublocalisation was identified if ECG-based origin either septal or free wall corresponded with the EPS origin and if on the horizontal plane was no significant mismatch as anterior/posterior (Fig. 2). Mismatches as anteriormid, posterior-mid were considered as non-significant mismatches.

3. For Joshi and Pytkowski algorithms that used nine zones in RVOT based on three vertical lines and three horizontal lines, match between the ECG and EPS origin was confirmed if ECG-based origin either septal or free wall corresponded with the EPS origin and if on the horizontal and vertical plane was no significant mismatch as anterior/posterior and inferior/ superior. Mismatches as anterior-mid, mid-posterior, superior-mid, mid-inferior were considered as non-significant mismatches.
The origin of RVOTA was established either on septal or on free wall when using the Ito algorithm (Fig. 1). Based on Dixit and Zhang algorithms, a more precise sublocalisation of RVOTA could be performed-septal vs free wall and on the horizontal plane (posterior, median and anterior zones) (Fig. 2). Using the Zhang or Pytkowski algorithm, location was identified in one of the nine regions of the RVOT divided by three vertical and three horizontal lines (Fig. 3).

One of the objectives of our study was to evaluate the concordance between the arrhythmia origin based on each ECG algorithm and the 3D mapping system when evaluated by both cardiologist and electrophysiologist. Next, we set out to establish any significant differences in terms of sensitivity and specificity when comparing RVOT ectopy localisation based on the five algorithms, performed by a general cardiologist versus an electrophysiologist. It is known that ECG electrodes' placement in both limb and precordial leads from standard 12-lead ECG may differ and lead to mistakes in correct localisation. So, evaluation was performed on the same ECG to avoid localisation discrepancies between cardiologist and electrophysiologist. Our objective was to determine if the use of complex, time-consuming analysis and difficult-to-memorise algorithms to predict a precise location (a region from one of the nine zones described by Pytkowski and Joshi) are feasible and preferable instead of determining a wider location of RVOT ectopies (septal or free wall location as in the Ito algorithm), when performed by a general cardiologist before the electrophysiologist evaluation.

\section{Electrophysiological procedure}

Procedures were performed in the fasting state, and all anti-arrhythmic drug therapy was discontinued at least five half-lives before. One quadripolar electrode catheter was inserted into a femoral vein and positioned in the right ventricle apex. An eight-French quadripolar catheter with a 4-mm distal electrode, sensor-enabled interelectrode spacing of 1-4-1 mm and a flexible tip (Abbott Laboratories, Abbott Park, IL, USA) was also inserted through the femoral vein in the right ventricle for mapping and ablation.

Twelve electrocardiographic leads and the bipolar intracardiac electrograms were recorded by optical disk. The filter settings for the intracardiac electrograms were set at $30-500 \mathrm{~Hz}$. Pacing was performed at twice the diastolic threshold with a programmable stimulator using stimuli with 2-ms duration. The 3D geometry of RVOT was constructed by navigating the mapping and ablation catheter within the RVOT using the non-contact electroanatomical mapping system (EnSite system, Abbott Laboratories). Areas of interest as the His bundle were 
Prisecaru et al. International Journal of Arrhythmia

(2021) $22: 22$

Page 4 of 10

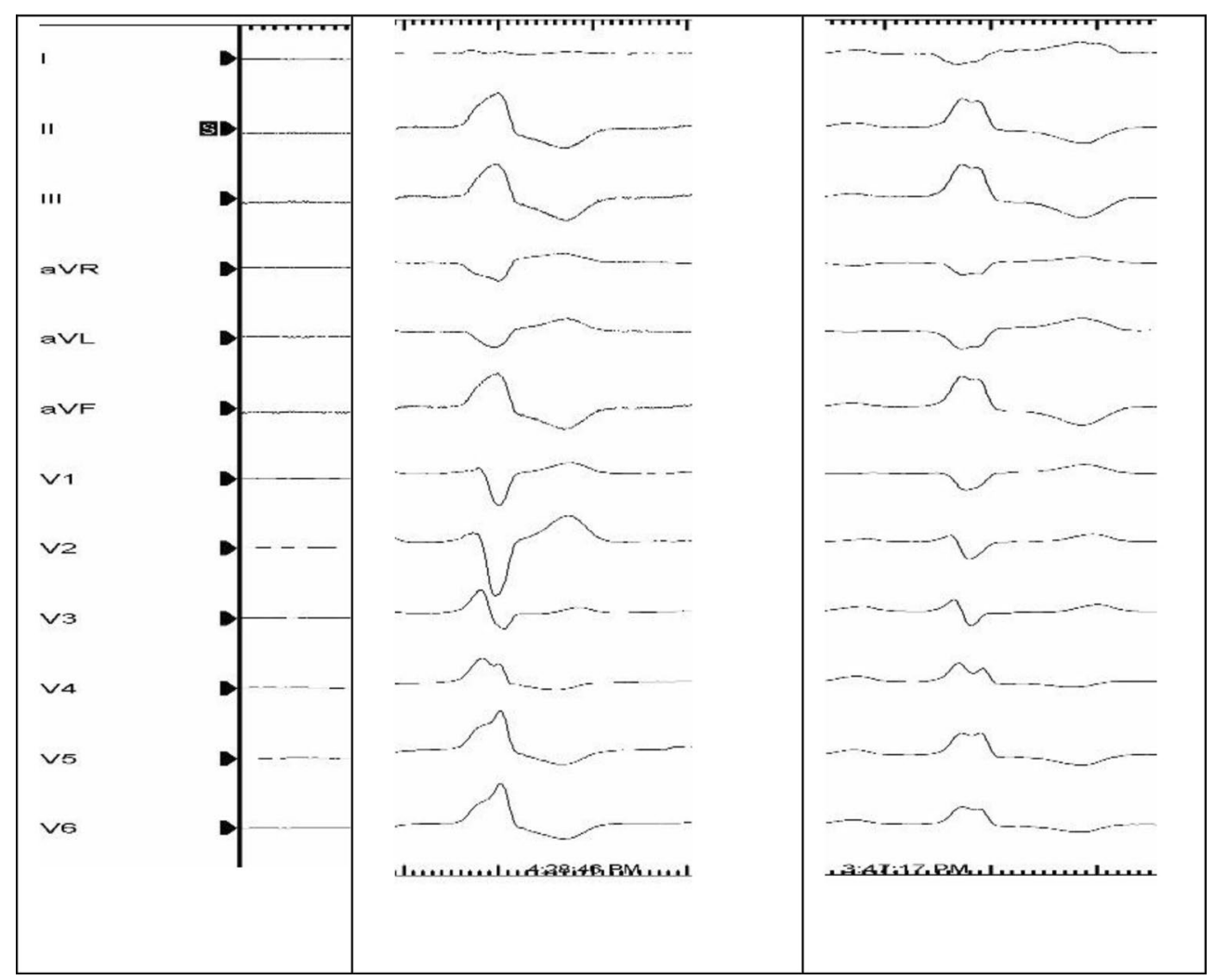

Fig. 1 Left and right panel ECG showing a septal vs free wall origin of the RVOT ectopy based on Ito algorithm. In both panels, PVC are characterised by a LBBB morphology with late transition in V4 and R/S ratio > 1 in lead II, III and AVF. The presence of QRS notching and the lower $R$ wave amplitude in the inferior leads is highly suggestive for a free wall origin as in the right panel. Absence of QRS notching and higher $R$ wave amplitude in the inferior leads suggest a septal origin of the PVC

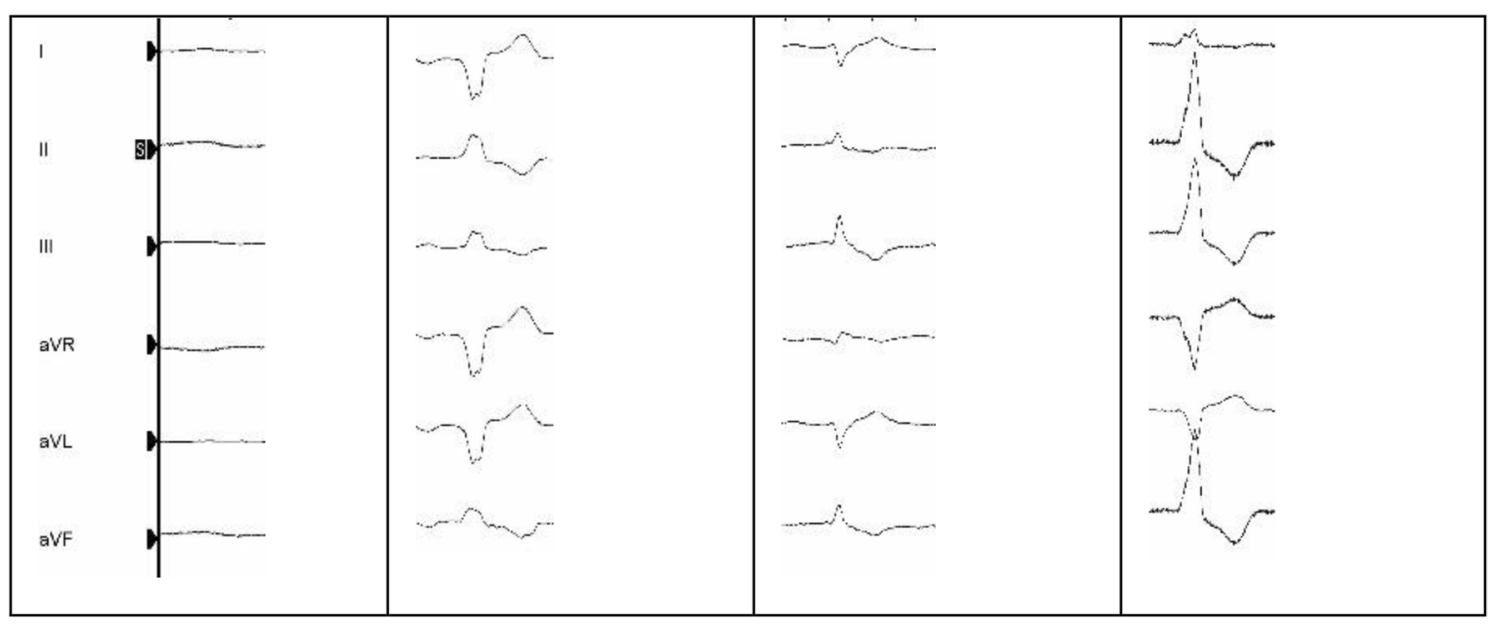

Fig. 2 Following Dixit and Chang algorithms, anterior, mid and posterior localisation (from left to right panel) in the RVOT can be established based on the differences of QRS morphology in lead I and AVL. QRS polarity in lead aV was assessed to determine the anterior or posterior arrhythmia locations. In the left panel, QS aspect in both lead I and AVL leads suggests an anterior location, while in the mid panel a $R / S$ ratio $<1$ and low $R$ wave amplitude in I and AVL leads favours a mid to anterior location of the PVC. In the right panel, a R/S ratio > 1 in lead I suggests a posterior origin 


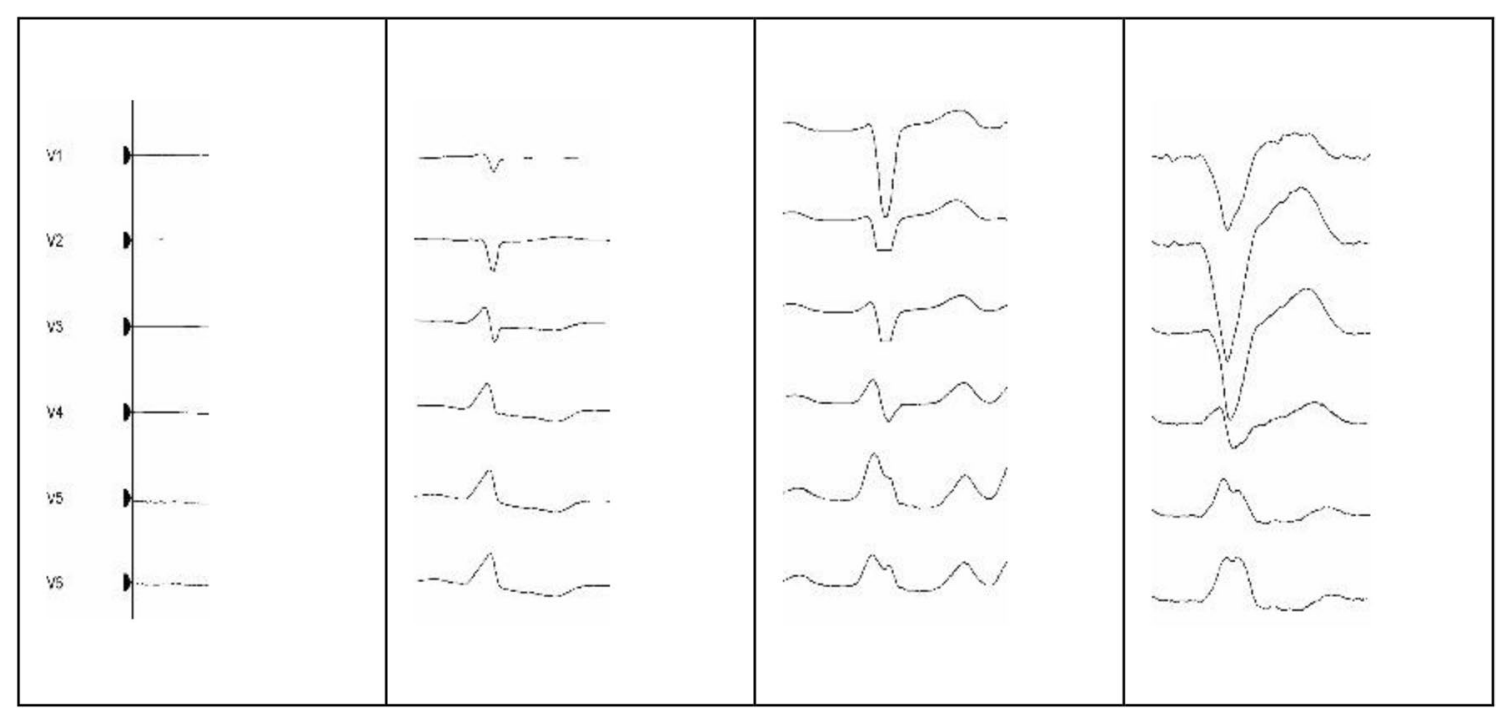

Fig. 3 ECG localization in the vertical plan of RVOT ectopies is based on the QRS morphology differences in precordial leads. A superior origin (left panel ECG) is characterised by $r<S$ in $\mathrm{V} 3, R>s$ in $\mathrm{V} 4$ ), a mid-origin is favoured by $r=s$ in $\mathrm{V} 4$ (mid panel ECG) and inferior localisation characterised by $r<\sin \mathrm{V} 4$ and $R$ in $\mathrm{V} 5$ (right panel ECG)

annotated using the 4-mm sensor-enabled tip catheter. Spontaneous PVC at baseline and during intravenous isoprenaline $(1-4 \mu \mathrm{g} / \mathrm{min})$ infusion was recorded.

For identification of earliest activation (EA) site, a broad colour setting with high-pass-filter set at $2 \mathrm{~Hz}$ was used. Then, the EA site was identified by stepping further back in time in which the red colour-zone shrinks down to the blue colour. Furthermore, unipolar virtual electrograms at this site were reconstructed. The presence of QS morphology was also used an additional criterion for EA site. Radiofrequency catheter ablation was targeted at the EA site. At each of the target sites, pace mapping was also performed to ensure at least 11 of 12 matching on the 12-lead ECG. Ablation was performed by delivering radiofrequency energy with the ablation catheter in temperaturecontrol mode (Stockert, Biosense Webster, Diamond Bar, CA, USA). The power output was titrated to as high as $40 \mathrm{~W}$ to achieve a target temperature $45^{\circ} \mathrm{C}$ for $60-120 \mathrm{~s}$. A waiting period of $30 \mathrm{~min}$ was applied to all patients. If complete elimination of VAs was not achieved, surrounding sites were further investigated by activation and pace mapping to find alternative ablation sites and subsequent ablations were applied to those sites. The ablation procedure was considered successful, if clinical PVC disappeared during RF, did not reoccur within $30 \mathrm{~min}$ after the last RF application and if PVCs were non-inducible after Isoproterenol infusion $(1-10 \mu \mathrm{g} / \mathrm{min})[29,30]$.

Based on the 3D electroanatomical non-contact mapping (Ensite, Abbott Laboratories, IL, USA), we divided RVOT through three vertical and three horizontal imaginary lines into nine distinct sites to facilitate the description of the origin of VPC based on the successful ablation site into nine subregions.

\section{Statistical analysis}

Data analysis was performed using IBM SPSS Statistics for Windows (version 20.0; IBM Corp., Armonk, NY, USA). Continuous variables are presented as the mean \pm standard deviation (SD). Categorical variables are expressed as counts and percentages. Coefficients of Kappa were used for determining the levels of interobserver agreement. Kappa values over 0.75 were accepted as excellent, $0.40-0.75$ and below 0.40 were accepted as fair to good and poor, respectively.

Table 1 Clinical characteristics

\begin{tabular}{ll}
\hline Age (years; mean \pm SD) & $47.74 \pm 17.93$ \\
Female/male ( $n)$ & $16 / 12$ \\
PVC burden (\%; mean \pm SD) & $18.51 \pm 9.62$ \\
LVEF (\%; mean \pm SD) & $69.1 \pm 11.5$ \\
Use of antiarrhythmic drugs (\%) & $39.28 \%$ \\
History of CHD, hypertension, diabetes, hypercolester- & $42.85 \%$ \\
olemia (\%) & \\
History of cardiomyopathy (\%) & $7.14 \%$
\end{tabular}

RVOT right ventricular outflow tract, $P V C$ premature ventricular contraction, LVEF left ventricular ejection fraction, $C H D$ coronary heart disease, $S D$ standard deviation 


\section{Results}

Twenty-eight patients with RVOTAs were enrolled into this study. Patient clinical characteristics are summarised in Table 1. Acute procedural success was achieved for all the included patients. Ablation was delivered at 22 septum sites and siz free wall sites.

The 3D electroanatomic maps were reconstructed for all patients, which were then applied to localise the origin of RVOTA ectopy recorded before the EPS and ablation procedure. The RVOTA chamber was correctly identified by the electrophysiologist in $28 / 28(100 \%)$ patients, and the sublocalisation within the RVOT was achieved in $20 / 28$ patients $(71.42 \%)(p=0.02)$ when using the first algorithm, 26/28(92.95\%) ( $p=0.35)$ when using the second algorithm, but with small discrepancies regarding the horizontal plane in $6 / 28$ (21.42\%). Following the third algorithm, correct location was identified in $24 / 28$ (85.71\%) $(p=0.23)$. Small discordances were observed in the vertical plane in $2 / 28(7.14 \%)$ and in the horizontal plane in $14 / 28$ patients $(50 \%)$. When using the fourth algorithm, correct sublocalisation was observed in 20/28 patients $(71.42 \%)(p=0.40)$ and mismatched regarding the horizontal plane in 10/28 patients (35.71\%). Following the fifth algorithm correct location was identified in $24 / 28$ patients $(85.71 \%)(p=0.23)$ and small mismatch in the horizontal plane was noticed in $6 / 28$ patients $(21.42 \%)$ and in the vertical plane in $3 / 28$ patients (10.71\%).

When ECGs were assessed by the general cardiologist, the sublocalisation within the RVOT was achieved in $18 / 28$ patients $(64.28 \%)(p=0.006)$ when using the first algorithm and 18/28(64.28\%) $(p=0.08)$ when using the second algorithm with small discrepancies regarding the horizontal plane in $2 / 28(7.14 \%)$. Following the third algorithm, correct location was identified in 14/28 (50\%) $(p=0.53)$. Small discordances were observed in the vertical plane in $5 / 28(17.85 \%)$ and in horizontal plane in $4 / 28$ patients $(14.28 \%)$. When using the fourth algorithm, correct sublocalisation was observed in $14 / 28$ patients $(50 \%)(p=0.0009)$ and mismatched regarding the horizontal plane in $5 / 28$ patients $(17.85 \%)$. Following the fifth algorithm, correct location was identified in $23 / 28$ patients $(82.14 \%)(p=0.14)$ and small mismatch in the horizontal plane was noticed in $4 / 28$ patients (14.28\%) and in the vertical plane in $4 / 28$ patients (14.28\%).

The five ECG algorithms were assessed for their diagnostic accuracy, specificity, sensitivity and likelihood ratios (LRs) of differentiating between the septal and free wall origin. The results are summarised in Table 2 . Assessment of the diagnostic accuracy showed that each of the five algorithms had only moderate accuracy, and the greatest accuracy was observed in the algorithm proposed by Pytkowski algorithm when assessed by a general cardiologist and Dixit algorithm when evaluated by the electrophysiologist. However, when the algorithms were compared for their accuracy, specificity, sensitivity, no significant differences were found $(p=0.99)$.

In addition, the receiver operating characteristic (ROC) curve analysis regarding the predictive value of $3 \mathrm{D}$ electroanatomical origin and the five ECG algorithms for differentiating the septal and free wall arrhythmias was conducted. The results showed a similar diagnostic accuracy among the five ECG algorithms, with areas under the curve (AUCs) ranging from 0.57 to 0.64 when assessed by an electrophysiologist and $0.54-0.63$ when evaluated by a general cardiologist $(p>0.5)$ (Fig. 4-panel A left, Fig. 5-right panel).

The interobserver agreement between the cardiologist and the electrophysiologists in localising between the septal and free wall was evaluated for each algorithm. The algorithm published by Ito had the highest kappa value of 0.77 , and acceptable kappa values of 0.55 and 0.4 were obtained for algorithms proposed by Pytkowski and Dixit, respectively. However, poor interobserver agreements were detected for algorithms proposed by Joshi and Zhang.

\section{Discussions}

All the five published 12-lead ECG algorithms for RVOTA differentiation were similar in terms of the diagnostic accuracy, specificity, sensitivity and LRs. The predicted accuracy of the ECG algorithms in our study

Table 2 Accuracy, sensitivity, specificity, positive and negative likelihood ratio ( $L R+$ and $L R-)$ of each algorithm evaluated by a cardiologist and, respectively, an electrophysiologist

\begin{tabular}{|c|c|c|c|c|c|}
\hline Alg & Accuracy & Sensitivity & Specificity & $\mathrm{LR}+$ & LR - \\
\hline 1 & $71.8 \%$ versus $75.86 \%$ & $68.75 \%$ versus $73.3 \%$ & $85.71 \%$ versus $85.71 \%$ & 4.81 versus 5.13 & 0.36 versus 0.31 \\
\hline 2 & $73.68 \%$ versus $90.32 \%$ & $73.33 \%$ versus $91.67 \%$ & $75 \%$ versus $85.71 \%$ & 2.93 versus 6.42 & 0.36 versus 0.1 \\
\hline 3 & $65.12 \%$ versus $84.5 \%$ & $61.11 \%$ versus $84.62 \%$ & $85.71 \%$ versus $85.71 \%$ & 4.28 versus 5.92 & 0.45 versus 0.18 \\
\hline 4 & $66.67 \%$ versus $75.86 \%$ & $68.75 \%$ versus $73.33 \%$ & $60 \%$ versus $85.71 \%$ & 1.72 versus 5.13 & 0.52 versus 0.31 \\
\hline 5 & $82.35 \%$ versus $84.85 \%$ & $81.48 \%$ versus $84.62 \%$ & $85.71 \%$ versus $85.71 \%$ & 5.77 versus 5.92 & 0.22 versus 0.18 \\
\hline
\end{tabular}




\section{ROC Plots}

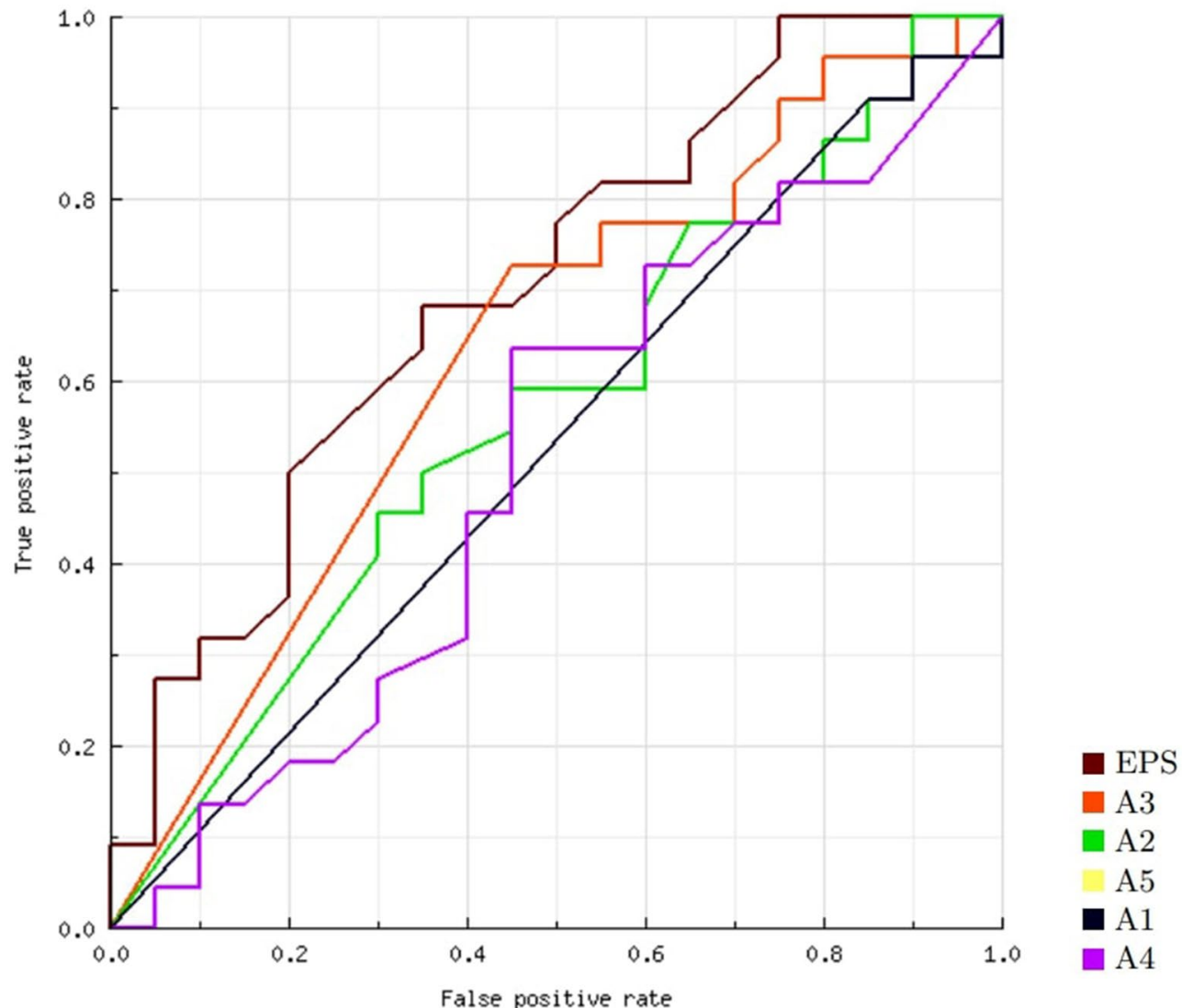

Fig. 4 Receiver operating characteristic (ROC) curve analysis regarding the predictive value of 3D electroanatomical origin (EPS) and the five ECG algorithms for differentiating (A1-A5) when assessment was performed by the cardiologist

ranged from 65.12 to $90.32 \%$ and the sensitivity ranged from 61.1 to $84.2 \%$, all of which were lower compared with results that were previously reported [9-18]. Possible explanations for the lack of reproducibility may be the differences in the population and the heterogeneity between the assessors in the present study and the developers of algorithms because two of the five ECG algorithms exhibited poor interobserver agreement-Joshi and Zhang.

The best interobserver agreement was observed with the algorithm published by Ito $(k-0.77)$. This could be explained by a wider sublocalisation of the RVOTA origin-septal or free wall. Algorithms with complex stepwise analysis and/or predicting a precise location are more prone to deliver inaccurate results and thus interobserver discrepancies.

When the general cardiologist assessed the ECGs, the Joshi algorithm revealed the lowest accuracy level $65.12 \%$ and lowest sensitivity $61.12 \%$. This could be explained partially by a more detailed design of the algorithm and a more refined sublocalisation of the RVOTA compared with algorithms differentiating only septal from free wall. The highest accuracy and sensitivity were achieved with the Pytkowski algorithm-82.35\% and 81.48\%, respectively, despite the more precise sublocalisation of RVOTA, but with an elementary algorithm design.

When ECGs were evaluated by the electrophysiologist, the lowest accuracy and sensitivity were observed for Ito and Zhang algorithms-75.86\% and $73.33 \%(p=0.9)$, respectively. Results are like those reported in other studies. Highest accuracy and sensitivity were achieved with the Dixit criteria's-90.32\% accuracy and $91.67 \%$ sensitivity, followed by the Pytkowski algorithm-84.85\% accuracy and $84.5 \%$ sensitivity $(p=0.91)$, slightly lower than those reported by the authors. Compared to Dixit algorithm, Pytkowski scheme leads to a more precise location of the RVOTA, not only in predicting septal vs free 


\section{ROC Plots}

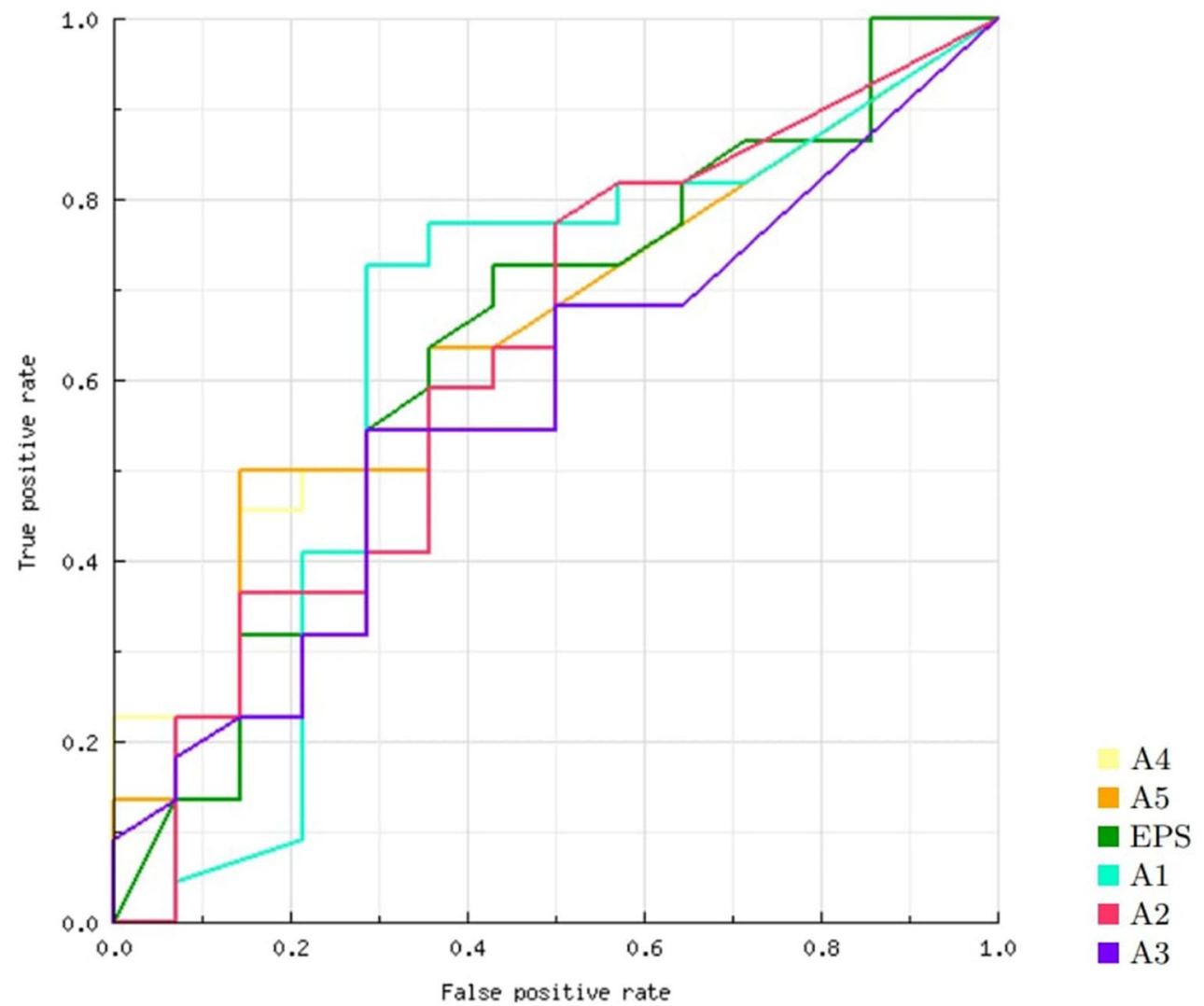

Fig. 5 Receiver operating characteristic (ROC) curve analysis regarding the predictive value of 3D electroanatomical origin (EPS) and the five ECG algorithms for differentiating (A1-A5) when assessment was performed by the electrophysiologist

wall location, but also in the horizontal and in the vertical planes.

When sublocalisation in one of the nine zones of the RVOT was intended, there was no significant difference in terms of accuracy and sensitivity between Pytkowski and Joshi schemes when assessment was performed by the general cardiologist $(p=0.82)$ or an electrophysiologist. Based on these findings and the acceptable kappa value of the Pytkowski algorithm (0.55), we can conclude that the use of a more detailed and difficult to memorise algorithm as Pytkowski can be routinely used to precisely localise the RVOTA origin instead of other algorithms with a less precise sublocalisation of RVOTA origin not only by general cardiologists but also for electrophysiologists.

The ECG-based algorithms for precise localising RVOTA origin may simplify the mapping process, reduce the procedural and fluoroscopic time and improve clinical outcomes, resulting in greater clinical utility.
Meanwhile, it is paramount to understand that the algorithms with a discriminating stepwise analysis may lessen the inaccurate results. Remembering detailed algorithms is not easy, and orientation of the subsequent steps may be challenging. Time-consuming and complex analyses of such algorithms may be more helpful while predicting the arrhythmia location and may lead to a better preparation before the procedure, but at the same time, the missed time in analysing the algorithm may be gained during the procedure. All the erroneous predictions of the ROTVA origin that used a 12-lead algorithm may result in inappropriate ablation approaches and negatively affect its effectiveness.

\section{Limitations}

The number of recruited patients is relatively small, and this may affect the results of the comparison among the ECG algorithms. Several limitations associated with using the 12-lead ECG algorithms were found in our 
study and have been reported in previous studies. Some algorithms require an accurate measurement of many ECG parameters, and thus, inconsistencies and heterogeneities between assessors are inevitable, due to lack of electronic callipers, wandering baselines [20-22]. Inaccuracies in ECG algorithms might also be caused by variable precordial lead placements or by the spatial relationship between the heart and torso [13, 31, 32]. As we did not perform magnetic resonance imaging (MRI) to all patients, right ventricular dysplasia cannot be ruled out. Therefore, the accuracy of algorithms cannot be correctly evaluated. The patients' age range was $15-67$ years for this study, and we did not include more paediatric and older patient populations; thus, the age-related changes in PVC morphology were not discussed.

\section{Conclusions}

All the five published 12-lead ECG algorithms for RVOTA differentiation were similar in terms of the diagnostic accuracy, specificity, sensitivity and LRs. In our study, when evaluated by both the electrophysiologist and cardiologist, the Pytkowski algorithm has the best accuracy and sensitivity among the evaluated algorithms, in predicting an even more precise location of the RVOTA origin, not only septal vs free wall, but also in the horizontal and in the vertical planes and an acceptable interobserver agreement.

\begin{abstract}
Abbreviations
RVOT: Right ventricular outflow tract; PVC: Premature ventricular complexes; RF: Radiofrequency; LBBB: Left bundle branch block; ECG: Electrocardiogram; RVOTA: Right ventricular outflow tract arrhythmias; EA: Earliest activation; ROC: Receiver operating characteristic; MRI: Magnetic resonance imaging; $V T$ : Ventricular tachycardia; LV: Left ventricle.
\end{abstract}

\section{Authors contribution}

RSP, CL collected the data and made ECG interpretation, all authors wrote and reviewed parts of the manuscript. All authors read and approved the final manuscript.

\section{Competing interest}

None.

\section{Author details}

${ }^{1}$ Cardiology Department, European Polisano Medlife Hospital, Izvorului Street 1A, 550172 Sibiu, Romania. ${ }^{2}$ Cardiology Department, Military Hospital Tunis, Tunis, Tunisia. ${ }^{3}$ Lucian Blaga University Sibiu, Victoriei Bd 10, 550024 Sibiu, Romania.

Received: 30 June 2021 Accepted: 24 September 2021 Published online: 01 December 2021

\footnotetext{
References

1. Valk SD, de Groot NM, Szili-Torok T, et al. Clinical characteristics and acute results of catheter ablation for outflow tract ventricular tachycardia or premature beats. J Interv Card Electrophysiol. 2012;35:301-9.

2. Brooks F, Burgess H. Idiopathic ventricular tachycardia. Medicine (Baltimore). 1988;67:271-94.
}

3. Joshi S, Wilber D. Ablation of idiopathic right ventricular outflow tract tachycardia-current perspectives. J Cardiovasc Electrophysiol. 2005;16:S52-8. https://doi.org/10.1111/j.1540-8167.

4. Ban JE, Park HC, Park JS, et al. Electrocardiographic and electrophysiological characteristics of premature ventricular complexes associated with left ventricular dysfunction in patients without structural heart disease. Europace. 2013;15:735-41.

5. Carballeira PL, Deyell MW, Frankel DS, Benhayon D, Squara F, Chik W, Kohari M, Deo R, Marchlinski FE. Ventricular premature depolarization QRS duration as a new marker of risk for the development of ventricular premature depolarization-induced cardiomyopathy. Heart Rhythm. 2014;11(2):299-306.

6. Del Carpio MF, Syed FF, Noheria A, Cha Y-M, Friedman PA, Hammill SC, Munger TM, Venkatachalam KL, Shen W-K, Packer DL, Asirvatham SJ. Characteristics of premature ventricular complexes as correlates of reduced left ventricular systolic function: study of the burden, duration, coupling interval, morphology and site of origin of PVCs. J Cardiovasc Electrophysiol. 2011;22(7):791-8.

7. Baman TS, Lange DC, Ilg KJ, Gupta SK, Liu T-Y, Alguire C, Armstrong W, Good E, Chugh A, Jongnarangsin K, Pelosi F, Crawford T, Ebinger M, Oral H, Morady F, Bogun F. Relationship between burden of premature ventricular complexes and left ventricular function. Heart Rhythm. 2010;7(7):865-9.

8. Callans DJ, Menz V, Schwartzman D, Gottlieb CD, Marchlinski FE. Repetitive monomorphic tachycardia from the left ventricular outflow tract: electrocardiographic patterns consistent with a left ventricular site of origin. J Am Coll Cardiol. 1997;29:1023-7. https://doi.org/10.1016/S07351097(97)00004-1.

9. Buxton AE, Waxman HL, Marchlinski FE, Simson MB, Cassidy D, Josephson ME. Right ventricular tachycardia: clinical and electrophysiological characteristics. Circulation. 1983;68:917-27. https://doi.org/10.1161/01.CIR. 68.5.917.

10. Jadonath RL, Schwartzman D, Preminger MW, Gottlieb CD, Marchlinski FE. Utility of 12-lead electrocardiogram in localizing the origin of right ventricular outflow tract tachycardia. Am Heart J. 1995;130:1107-13. https:// doi.org/10.1016/0002-8703(95)90215-5.

11. Movsowitz C, Schwartzman D, Callans DJ, Preminger M, Zado E, Gottlieb CD, Marchlinski FE. Idiopathic right ventricular tachycardia: narrowing the anatomic location for successful ablation. Am Heart J. 1996;131:930-6. https://doi.org/10.1016/S0002-8703(96)90175-1.

12. Priori SG, Blomstrom-Lundqvist C, Mazzanti A, Blom N, et al. 2015 ESC Guidelines for the management of patients with ventricular arrhythmias and the prevention of sudden cardiac death. Eur Heart J. 2015;36:2841-2.

13. Morady F, Kadish AH, DiCarlo L, Kou WH, Winston S, deBuitlier M, Calkins $H$, Rosenheck S, Sousa J. Long-term results of catheter ablation of idiopathic right ventricular tachycardia. Circulation. 1990;82:2093-9.

14. Tada H, Hiratsuji T, Naito S, Kurosaki K, Ueda M, Ito S, Shinbo G, Hoshizaki H, Oshima S, Nogami A, Taniguchi K. Prevalence and characteristics of idiopathic outflow tract tachycardia with QRS alteration following catheter ablation requiring additional radiofrequency ablation at a different point in the outflow tract. Pacing Clin Electrophysiol. 2004;27:1240-9.

15. Yamada T, McElderry HT, Doppalapudi H, Murakami Y, Yoshida Y, Yoshida N, Okada T, Tsuboi N, Inden Y, Murohara T, Epstein AE, Plumb VJ, Singh SP, Kay GN. Idiopathic ventricular arrhythmias originating from the aortic root prevalence, electrocardiographic and electrophysiologic characteristics, and results of radiofrequency catheter ablation. J Am Coll Cardiol. 2008;52:139-47.

16. Betensky BP, Park RE, Marchlinski FE, et al. The V(2) transition ratio: a new electrocardiographic criterion for distinguishing left from right ventricular outflow tract tachycardia origin. J Am Coll Cardiol. 2011;57:2255-62.

17. Cheng Z, Cheng K, Deng H, et al. The R-wave deflection interval in lead V3 combining with R-wave amplitude index in lead V1: a new surface ECG algorithm for distinguishing left from right ventricular outflow tract tachycardia origin in patients with transitional lead at V3. Int J Cardiol. 2013;168:1342-8.

18. Igarashi $M$, Nogami $A$, Sekiguchi $Y$, et al. The $Q R S$ morphology pattern in V5R is a novel and simple parameter for differentiating the origin of idiopathic outflow tract ventricular arrhythmias. Europace. 2015;17:1107-16.

19. Ito $\mathrm{S}$, Tada H, Naito $\mathrm{S}$, et al. Development and validation of an ECG algorithm for identifying the optimal ablation site for idiopathic ventricular outflow tract tachycardia. J Cardiovasc Electrophysiol. 2003;14:1280-6. 
20. Nakano M, Ueda M, Ishimura M, et al. Estimation of the origin of ventricular outflow tract arrhythmia using synthesized right-sided chest leads. Europace. 2014;16:1373-8.

21. Ouyang F, Fotuhi P, Ho SY, et al. Repetitive monomorphic ventricular tachycardia originating from the aortic sinus cusp: electrocardiographic characterization for guiding catheter ablation. J Am Coll Cardiol. 2002:39:500-8

22. Yoshida $\mathrm{N}$, Inden $\mathrm{Y}$, Uchikawa T, et al. Novel transitional zone index allows more accurate differentiation between idiopathic right ventricular outflow tract and aortic sinus cusp ventricular arrhythmias. Heart Rhythm. 2011:8:349-56.

23. Yoshida N, Yamada T, McElderry HT, et al. A novel electrocardiographic criterion for differentiating a left from right ventricular outflow tract tachycardia origin: the V2S/N3R index. J Cardiovasc Electrophysiol. 2014;25:747-53.

24. Zhang F, Chen M, Yang B, et al. Electrocardiographic algorithm to identify the optimal target ablation site for idiopathic right ventricular outflow tract ventricular premature contraction. Europace. 2009;11:1214-20.

25. Hutchinson MD, Garcia FC. An organized approach to the localization, mapping, and ablation of outflow tract ventricular arrhythmias. J Cardiovasc Electrophysiol. 2013;24(10):1189-97.
26. Dixit S, Gerstenfeld EP, Callans DJ, Marchlinski FE. Electrocardiographic patterns of superior right ventricular outflow tract tachycardias distinguishing septal and free-wall wits of origin. J Cardiovasc Electrophys. 2003;14:1-7. https://doi.org/10.1046/j.1540-8167.2003.02404.x.

27. Pytkowski M, Maciąg A, Sterliński M, et al. Novel algorithm for arrhythmogenic focus localization in patients with right ventricular outflow tract arrhythmias. Cardiol J. 2014;21(3):284-92.

28. Rillig A, Lin T, Ouyang F, Kuck KH, Tilz RR. Which is the appropriate arrhythmia burden to offer RF ablation For RVOT tachycardias? J Atr Fibrillation. 2014;7(4):1157.

29. Issa ZF, Miller JM, DP Zipes. Adenosine-sensitive (outflow tract) ventricular tachycardia. In: Clinical Arrhythmology and Electrophysiology. A companion to Braunwald's Heart Disease. 2009; pp. 562-586.

30. Fuenmayor A. Treatment or cure of right ventricular outflow tract tachycardia. J Atr Fibrillation. 2014;7(1):1038.

31. Zhou X, Fang L, Wang Z, Liu H, Mao W. Comparative analysis of electrocardiographic imaging and ECG in predicting the origin of outflow tract ventricular arrhythmias. J Int Med Res. 2020;48(3):131-2.

32. Jamil-Copley S, Bokan R, Kojodjojo P, Qureshi N, Koa-Wing M, Hayat S, et al. Noninvasive electrocardiographic mapping to guide ablation of outflow tract ventricular arrhythmias. Heart Rhythm. 2014;11(4):587-94.
Ready to submit your research? Choose BMC and benefit from:

- fast, convenient online submission

- thorough peer review by experienced researchers in your field

- rapid publication on acceptance

- support for research data, including large and complex data types

- gold Open Access which fosters wider collaboration and increased citations

- maximum visibility for your research: over 100M website views per year

At BMC, research is always in progress.

Learn more biomedcentral.com/submissions 